\title{
Simulation for Clinical Repeated-Dose Pharmacokinetic Trials Applying a Peak-and-Trough Sampling Design to Estimate Oral Clearance
}

\author{
Kazuya IshidA, Yuichiro KaYano, Masato TAguchi, and Yukiya Hashimoto* \\ Graduate School of Pharmaceutical Sciences, University of Toyama; 2630 Sugitani, Toyama 930-0194, Japan. \\ Received June 22, 2007; accepted September 7, 2007; published online September 7, 2007
}

We performed a simulation for the clinical pharmacokinetic study, in which blood was sampled at two time points corresponding to the peak concentration $\left(C_{\text {peak }}\right)$ and trough concentration $\left(C_{\text {trough }}\right)$ following repetitive oral drug administration to subjects. We estimated the approximate oral clearance $\left(C L / F_{\text {approx }}\right)$ as $2 \cdot D /\left(C_{\text {peak }} \cdot \tau+C_{\text {trough }} \cdot \tau\right)$, where $D$ is the dose, and $\tau$ is the dosing interval. The $C L / F_{\text {approx }}$ value was accurate for drugs with a long-elimination half-life, and the estimation error of the $C L / F$ value was slightly increased for drugs with a shorter elimination half-life. The accuracy of $C L / F_{\text {approx }}$ in each subject was not affected by the magnitude of the interindividual pharmacokinetic variability, but was significantly decreased by the larger measurement error of drug concentrations (or intraindividual pharmacokinetic variability). We further performed several computer simulations to mimic statistical hypothesis testing following the clinical repeated-dose pharmacokinetic trials. The statistical power to detect the difference of oral clearance between two groups was marginally dependent on the measurement error of drug concentration, but was highly dependent on the interindividual pharmacokinetic variability. These findings suggested that the peak-and-trough sampling design to estimate the $C L / F_{\text {approx }}$ value is useful for clinical repeated-dose pharmacokinetic trials, and that the study design and protocol should be evaluated carefully by computer simulation prior to a real clinical trial.

Key words clinical pharmacokinetic trial; oral clearance; sampling design; steady-state simulation

The clinical pharmacologists are charged with the obligation to quantify the dose-response relationship (pharmacokinetics and pharmacodynamics) of clinically useful drugs. ${ }^{1)}$ Clinical pharmacokinetic studies are performed to determine the appropriate use of medicines according to patient characteristics, such as the disease and genotype of drug-metabolizing enzymes, and to predict the influence of pharmacokinetic drug interactions. ${ }^{2-7)}$ The area under the concentration-time curve $(A U C)$ and/or oral clearance $(C L / F)$ of orally administered drugs is a key pharmacokinetic parameter to evaluate drug exposure in the patient population. In the present study, we evaluated the usefulness of clinical repeated-dose pharmacokinetic trials, applying a simple peak-and-trough sampling design in order to estimate the $C L / F$ value. That is, blood was sampled at two points corresponding to the peak concentration $\left(C_{\text {peak }}\right)$ and trough concentration $\left(C_{\text {trough }}\right)$ following repetitive oral drug administration to a patient (Fig. $1)$. The approximate $C L / F\left(C L / F_{\text {approx }}\right)$ value in the patient

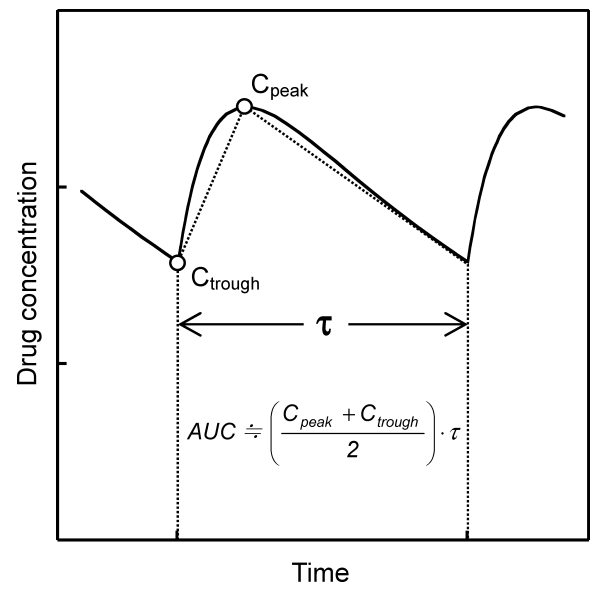

Fig. 1. Approximate Estimation of $A U C$ by Measuring $C_{\text {peak }}$ and $C_{\text {trough }}$ Following Clinical Practice-Resembling Repetitive Dosing may be estimated by the following equations:

$$
\begin{aligned}
& A U C \fallingdotseq\left(\frac{C_{\text {peak }}+C_{\text {trough }}}{2}\right) \cdot \tau \\
& C L / F_{\text {approx }}=\frac{D}{A U C}=\frac{2 \cdot D / \tau}{C_{\text {peak }}+C_{\text {trough }}}
\end{aligned}
$$

where $D$ is the dose, and $\tau$ is the dosing interval. The specific purpose of the present study was to investigate the accuracy of the $C L / F_{\text {approx }}$ value estimated by the peak-and-trough sampling design. We further performed several computer simulations to mimic statistical hypothesis testing following a clinical practice-resembling repeated-dose pharmacokinetic trial.

\section{MATERIALS AND METHODS}

Evaluation of Accuracy of $C L / F_{\text {approx }}$ Estimated by $\boldsymbol{C}_{\text {peak }}$ and $\boldsymbol{C}_{\text {trough }}$ To evaluate the accuracy of the $C L / F_{\text {approx }}$ value for various hypothetical drugs, we assumed a 1-compartment model with first-order input, and calculated the ratio of $C L / F_{\text {approx }}$ to true oral clearance $(C L / F)$ as follows:

$$
\begin{aligned}
\frac{C L / F_{\text {approx }}}{C L / F}= & \frac{2 \cdot D / \tau}{k_{\mathrm{e}} \cdot V / F} \cdot\left(C_{\text {peak }}+C_{\text {trough }}\right)^{-1} \\
= & \frac{2 \cdot\left(k_{\mathrm{a}}-k_{\mathrm{e}}\right)}{k_{\mathrm{e}} \cdot k_{\mathrm{a}} \cdot \tau} \cdot\left\{\frac{\exp \left(-k_{\mathrm{e}} \cdot t_{\max }\right)+\exp \left(-k_{\mathrm{e}} \cdot \tau\right)}{1-\exp \left(-k_{\mathrm{e}} \cdot \tau\right)}\right. \\
& \left.-\frac{\exp \left(-k_{\mathrm{a}} \cdot t_{\max }\right)+\exp \left(-k_{\mathrm{a}} \cdot \tau\right)}{1-\exp \left(-k_{\mathrm{a}} \cdot \tau\right)}\right\}^{-1}
\end{aligned}
$$

where $V / F$ is the apparent volume of distribution, $k_{\mathrm{e}}$ is the elimination rate constant, $k_{\mathrm{a}}$ is the absorption rate constant, and $t_{\max }$ is the time to reach maximal drug concentration after the last dose, described as follows: 
Table 1. Accuracy of $C L / F_{\text {approx }}$ for Hypothetical Drugs with Various $k_{\mathrm{e}}$ and $k$

\begin{tabular}{|c|c|c|c|c|c|}
\hline \multirow{2}{*}{$\begin{array}{c}t_{1 / 2, k_{\mathrm{e}}}{ }^{a)} \\
(\mathrm{h})\end{array}$} & \multirow{2}{*}{$\begin{array}{c}t_{1 / 2, k_{\mathrm{a}}} k^{b} \\
(\mathrm{~h})\end{array}$} & \multirow{2}{*}{$\begin{array}{c}\tau \\
(\mathrm{h})\end{array}$} & \multirow{2}{*}{$\begin{array}{l}t_{\max } \\
\text { (h) }\end{array}$} & $C_{\text {peak }}$ & \multirow{2}{*}{$\frac{C L / F_{\text {approx }}}{C L / F}$} \\
\hline & & & & $C_{\text {trough }}$ & \\
\hline 48 & $1 / 3$ & 12 & 1.517 & 1.155 & 1.009 \\
\hline 48 & $2 / 3$ & 12 & 2.379 & 1.133 & 1.017 \\
\hline 48 & 1 & 12 & 2.996 & 1.115 & 1.020 \\
\hline 48 & 2 & 12 & 4.082 & 1.078 & 1.020 \\
\hline 48 & 3 & 12 & 4.612 & 1.056 & 1.016 \\
\hline 24 & $1 / 3$ & 12 & 1.487 & 1.336 & 1.009 \\
\hline 24 & $2 / 3$ & 12 & 2.331 & 1.285 & 1.017 \\
\hline 24 & 1 & 12 & 2.936 & 1.245 & 1.020 \\
\hline 24 & 2 & 12 & 4.006 & 1.162 & 1.020 \\
\hline 24 & 3 & 12 & 4.531 & 1.116 & 1.016 \\
\hline 12 & $1 / 3$ & 12 & 1.430 & 1.790 & 1.005 \\
\hline 12 & $2 / 3$ & 12 & 2.238 & 1.660 & 1.025 \\
\hline 12 & 1 & 12 & 2.821 & 1.558 & 1.034 \\
\hline 12 & 2 & 12 & 3.859 & 1.355 & 1.037 \\
\hline 12 & 3 & 12 & 4.373 & 1.248 & 1.031 \\
\hline 8 & $1 / 3$ & 12 & 1.376 & 2.405 & 0.990 \\
\hline 8 & $2 / 3$ & 12 & 2.150 & 2.152 & 1.023 \\
\hline 8 & 1 & 12 & 2.710 & 1.957 & 1.041 \\
\hline 8 & 2 & 12 & 3.716 & 1.583 & $1.052^{c)}$ \\
\hline 8 & 3 & 12 & 4.218 & 1.397 & 1.044 \\
\hline 4 & $1 / 3$ & 12 & 1.234 & 5.919 & $0.892^{c)}$ \\
\hline 4 & $2 / 3$ & 12 & 1.914 & 4.783 & 0.970 \\
\hline 4 & 1 & 12 & 2.411 & 3.957 & 1.020 \\
\hline 4 & 2 & 12 & 3.321 & 2.530 & $1.072^{c)}$ \\
\hline 4 & 3 & 12 & 3.786 & 1.945 & $1.071^{c)}$ \\
\hline
\end{tabular}

a) $t_{1 / 2, k_{\mathrm{c}}}=0.693 / k_{\mathrm{e}}$. b) $t_{1 / 2, k_{\mathrm{a}}}=0.693 / k_{\mathrm{a}}$. c) Errors are more than $5 \%$.

$$
t_{\max }=\frac{1}{k_{\mathrm{a}}-k_{\mathrm{e}}} \cdot \ln \left\{\frac{k_{\mathrm{a}}}{k_{\mathrm{e}}} \cdot \frac{1-\exp \left(-k_{\mathrm{e}} \cdot \tau\right)}{1-\exp \left(-k_{\mathrm{a}} \cdot \tau\right)}\right\}
$$

Table 1 summarizes the $t_{1 / 2, k_{\mathrm{e}}}\left(=0.693 / k_{\mathrm{e}}\right), t_{1 / 2, k_{\mathrm{a}}}\left(=0.693 / k_{\mathrm{a}}\right)$, $\tau$, and $t_{\max }$ values of various hypothetical drugs evaluated in the present study.

We further evaluated the effect of the measurement error of $C_{\text {peak }}$ and $C_{\text {trough }}$ (or intraindividual pharmacokinetic variability) on the accuracy of the $C L / F_{\text {approx }}$ value. It is possible that interindividual pharmacokinetic variability is also responsible for the measurement error of the $C_{\text {peak }}$, because we do not know the true $t_{\max }$ in a subject before blood sampling. Therefore, we assumed typical hypothetical drugs with a mean $t_{1 / 2, k_{\mathrm{a}}}$ of $1 \mathrm{~h}, C L / F$ of $1, V / F$ of $12 / 0.693, t_{1 / 2, k_{\mathrm{e}}}$ of $12 \mathrm{~h}$, and $t_{\max }$ of $2.821 \mathrm{~h}$ (Table 1), and with various inter- and intraindividual pharmacokinetic variability. The $k_{\mathrm{a}}$ value of a drug in the $i$-th individual $\left(k_{\mathrm{a} i}\right)$ is described as follows:

$$
k_{\mathrm{a} i}=0.693 \cdot \exp \left(\eta_{k_{\mathrm{a}}}\right)
$$

where $\eta_{k_{i j}}$ is a random variable distributed normally with a mean of zero and variance of $\omega_{k_{\mathrm{a}}}^{2}$. Similarly, the $k_{\mathrm{e}}$ value of the drug in the $i$-th individual $\left(k_{\mathrm{e} i}\right)$ is described as follows:

$$
\begin{aligned}
& k_{\mathrm{e} i}=\frac{C L / F_{i}}{V / F_{i}} \\
& C L / F_{i}=1 \cdot \exp \left(\eta_{C L / F_{i}}\right) \\
& V / F_{i}=\frac{12}{0.693} \cdot \exp \left(\eta_{V / F_{i}}\right)
\end{aligned}
$$

where $C L / F_{i}$ is oral clearance in the $i$-th individual, and $\eta_{C L / F}$

\begin{tabular}{|c|c|c|c|c|c|c|}
\hline \multirow{2}{*}{ Trial } & \multirow{2}{*}{$n$} & \multirow{2}{*}{$C L / F$} & \multirow{2}{*}{$\omega, \sigma^{a}$} & \multicolumn{3}{|c|}{ Statistical significance } \\
\hline & & & & N.S. ${ }^{b)}$ & $p<0.05$ & $p<0.01$ \\
\hline \multirow[t]{2}{*}{1} & 20 & 1.0 & B & $6 / 30$ & $10 / 30$ & $14 / 30$ \\
\hline & 20 & 0.7 & B & & & \\
\hline \multirow[t]{2}{*}{2} & 20 & 1.0 & $\mathrm{C}$ & $10 / 30$ & $11 / 30$ & $9 / 30$ \\
\hline & 20 & 0.7 & $\mathrm{C}$ & & & \\
\hline \multirow[t]{2}{*}{3} & 20 & 1.0 & D & $11 / 30$ & $9 / 30$ & $10 / 30$ \\
\hline & 20 & 0.7 & D & & & \\
\hline \multirow[t]{2}{*}{4} & 20 & 1.0 & $\mathrm{E}$ & $2 / 30$ & $2 / 30$ & $26 / 30$ \\
\hline & 20 & 0.7 & $\mathrm{E}$ & & & \\
\hline \multirow[t]{2}{*}{5} & 20 & 1.0 & $\mathrm{~F}$ & $13 / 30$ & $8 / 30$ & $9 / 30$ \\
\hline & 20 & 0.7 & $\mathrm{~F}$ & & & \\
\hline \multirow[t]{2}{*}{6} & 30 & 1.0 & B & $13 / 30$ & $10 / 30$ & $7 / 30$ \\
\hline & 10 & 0.7 & B & & & \\
\hline \multirow[t]{3}{*}{7} & 10 & 1.3 & B & & & \\
\hline & 20 & 1.0 & B & $5 / 30$ & $6 / 30$ & $19 / 30$ \\
\hline & 10 & 0.7 & B & & & \\
\hline
\end{tabular}
is the random variable distributed normally with a mean of
Table 2. The $\omega$ and $\sigma$ Values of Hypothetical Drugs ${ }^{2-8)}$

\begin{tabular}{lllllll}
\hline \hline & $\mathrm{A}$ & $\mathrm{B}$ & $\mathrm{C}$ & $\mathrm{D}$ & $\mathrm{E}$ & $\mathrm{F}$ \\
\hline$\omega_{C L / F}$ & 0.4 & 0.4 & 0.4 & 0.4 & 0.3 & 0.5 \\
$\omega_{V / F}$ & 0.4 & 0.4 & 0.4 & 0.4 & 0.3 & 0.5 \\
$\omega_{k_{\mathrm{a}}}$ & 0.8 & 0.8 & 0.8 & 0.8 & 0.6 & 1.0 \\
$\sigma_{3}$ & 0.05 & 0.1 & 0.15 & 0.2 & 0.1 & 0.1 \\
$\sigma_{12}$ & 0.05 & 0.1 & 0.15 & 0.2 & 0.1 & 0.1 \\
\hline
\end{tabular}

Table 3. Protocols and Results of Various In-Silico Clinical Trials

a) See Table 2. b) Not significant.

zero and variance of $\omega_{C L / F}^{2} V / F_{i}$ is the apparent volume of distribution in the $i$-th individual, and $\eta_{V / F_{i}}$ is the random variable distributed normally with a mean of zero and variance of $\omega_{V / F}^{2}$. We assumed that the sampling time is $3 \mathrm{~h}$ and $12 \mathrm{~h}$ after the twice-daily dose $(\tau=12 \mathrm{~h})$ for all subjects. Thus, drug concentrations at $3 \mathrm{~h}\left(C_{3 i}\right)$ and $12 \mathrm{~h}\left(C_{12 i}\right)$ after the last dose in the $i$-th individual are described as follows:

$$
\begin{aligned}
C_{3 i}= & \frac{D_{i} \cdot k_{\mathrm{a} i}}{V / F_{i} \cdot\left(k_{\mathrm{a} i}-k_{\mathrm{e} i}\right)} \\
& \cdot\left\{\frac{\exp \left(-k_{\mathrm{e} i} \cdot 3\right)}{1-\exp \left(-k_{\mathrm{e} i} \cdot 12\right)}-\frac{\exp \left(-k_{\mathrm{a} i} \cdot 3\right)}{1-\exp \left(-k_{\mathrm{a} i} \cdot 12\right)}\right\} \cdot \exp \left(\varepsilon_{3 i}\right) \\
C_{12 i}= & \frac{D_{i} \cdot k_{\mathrm{a} i}}{V / F_{i} \cdot\left(k_{\mathrm{a} i}-k_{\mathrm{e} i}\right)} \\
& \cdot\left\{\frac{\exp \left(-k_{\mathrm{e} i} \cdot 12\right)}{1-\exp \left(-k_{\mathrm{e} i} \cdot 12\right)}-\frac{\exp \left(-k_{\mathrm{a} i} \cdot 12\right)}{1-\exp \left(-k_{\mathrm{a} i} \cdot 12\right)}\right\} \cdot \exp \left(\varepsilon_{12 i}\right)
\end{aligned}
$$

where $D_{i}$ is the dose in the $i$-th individual, and $\varepsilon_{3 i}$ and $\varepsilon_{12 i}$ are the random variables distributed normally with means of zero and variance of $\sigma_{3}^{2}$ and $\sigma_{12}^{2}$, respectively. Finally, $C L / F_{\text {approx }}$ in the $i$-th individual $\left(C L / F_{\text {approx }, i}\right)$ is described as follows:

$$
C L / F_{\text {approx }, i}=\frac{2 \cdot D_{i} / 12}{C_{3 i}+C_{12 i}}
$$

The accuracy of $C L / F_{\text {approx }, i}$ was evaluated by examining the correlation between $C L / F_{i}$ and $C L / F_{\text {approx }, i}$ in 50 subjects. Table 2 summarizes the $\omega$ and $\sigma$ values of various hypothetical drugs evaluated in the present study. ${ }^{2-8)}$

Simulation for Statistical Hypothesis Testing We further performed several computer simulations to mimic statis- 
tical hypothesis testing following the clinical repeated-dose pharmacokinetic trial. We assumed a hypothetical drug with a mean $t_{1 / 2, k_{\mathrm{a}}}$ of $1 \mathrm{~h}$ and $V / F$ of $12 / 0.693$. The other characteristics of the test drug, the number of subject groups, and the number of subjects in each group $(n)$ are summarized in Table 3. $C L / F_{\text {approx }, i}$ in each subject was calculated as described above, and the difference in the mean oral clearance between two groups was evaluated by Student's $t$-test. If the number of groups was three, we used one-way analysis of variance (ANOVA).

\section{RESULTS AND DISCUSSION}

We first evaluated the accuracy of $C L / F_{\text {approx }}$ for hypothetical drugs with various $k_{\mathrm{a}}$ and $k_{\mathrm{e}}$ (Table 1 ). In the present study, $t_{1 / 2, k_{\mathrm{a}}}$ values $\left(0.693 / k_{\mathrm{a}}\right)$ were assumed to range from $1 / 3 \mathrm{~h}$ to $3 \mathrm{~h}$, and $\tau$ was fixed at $12 \mathrm{~h}$ for all hypothetical drugs. The $t_{\max }$ values ranged from 1.517 to $4.612 \mathrm{~h}$ for drugs with $t_{1 / 2, k_{\mathrm{e}}}\left(0.693 / k_{\mathrm{e}}\right)$ of $48 \mathrm{~h}$, and the ratios of $C L / F_{\text {approx }}$ to $C L / F$ were almost identical to 1 , indicating that $C L / F_{\text {approx }}$ values were estimated very accurately (Table 1 ). In addition, it is apparently conceivable that $C_{\text {peak }}$ may be close to $C_{\text {trough }}$ for drugs with long $t_{1 / 2, k_{\mathrm{e}}}$ relative to $\tau$, and that the $A U C$ value for
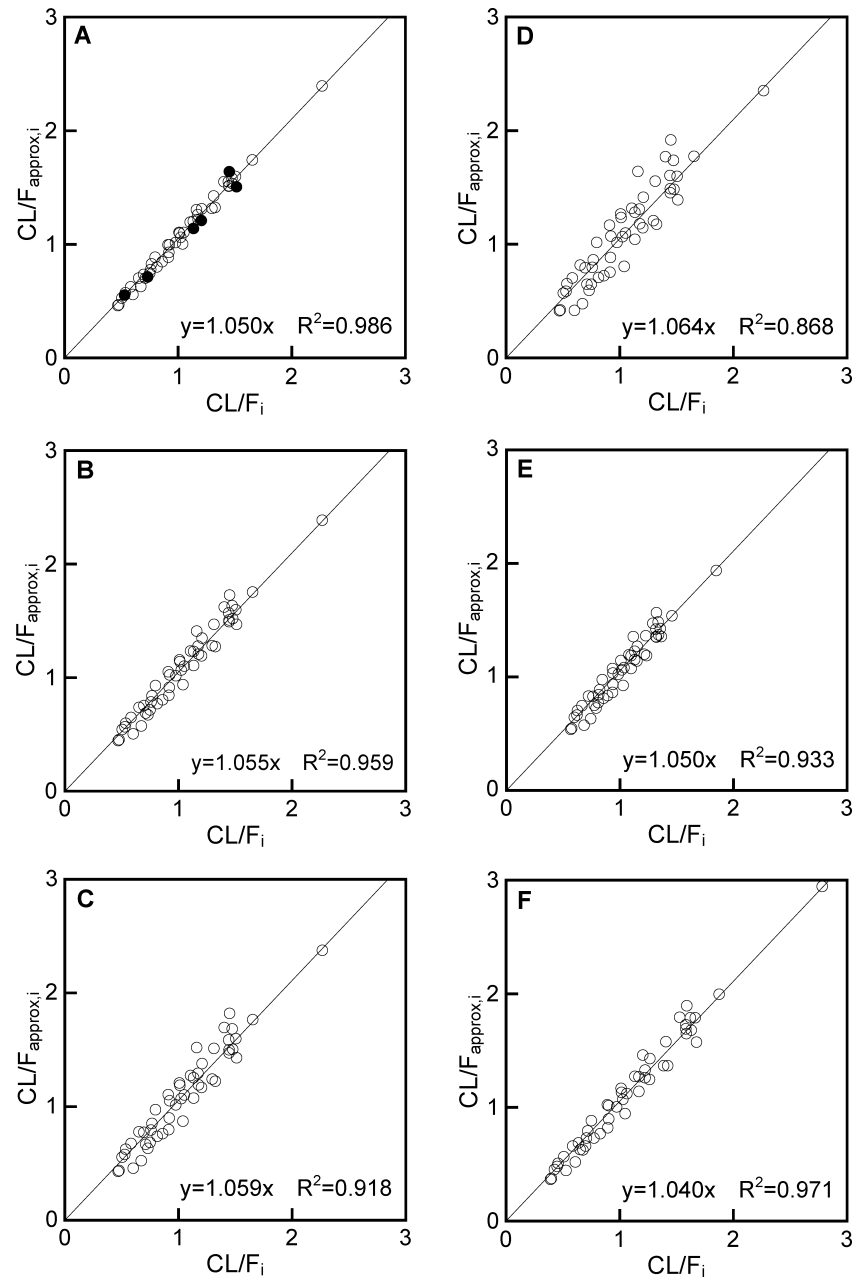

Fig. 2. Accuracy of $C L / F_{\text {approx }, i}$ for Hypothetical Drugs with Various $\omega$ and $\sigma$

The $\omega$ and $\sigma$ values for $\mathrm{A}-\mathrm{F}$ are shown in Table 2. Closed circles in A indicate 6 typical subjects whose blood drug concentrations are shown in Fig. 3. the drug may be nearly equal to $C_{\text {trough }} \cdot \tau$. However, the $C_{\text {peak }} / C_{\text {trough }}$ values were not always nearly equal to 1 even in the drug with $t_{1 / 2, k_{\mathrm{e}}}$ of $48 \mathrm{~h}$ (Table 1 ). Therefore, the peak-andtrough sampling design is preferable to the single-trough sampling design especially for the drug with short $t_{1 / 2, k_{\mathrm{a}}}$. Furthermore, the accuracy of $C L / F_{\text {approx }}$ was also fairly good for drugs with $t_{1 / 2, k_{\mathrm{e}}}$ values of $24 \mathrm{~h}, 12 \mathrm{~h}$, and $8 \mathrm{~h}$ (Table 1). On the other hand, the ratios of $C L / F_{\text {approx }}$ to $C L / F$ ranged from 0.892 to 1.072 for the drugs with $t_{1 / 2, k_{c}}$ of $4 \mathrm{~h}$. It should be noted that we should not use $C L / F_{\text {approx }}$ without extreme caution for drugs with very short $t_{1 / 2, k_{\mathrm{c}}}$ relative to $\tau$.

We next evaluated the accuracy of $C L / F_{\text {approx }}$ for hypothetical drugs with various $\omega$ and $\sigma$ values. We assumed drugs with a mean $t_{1 / 2, k_{\mathrm{a}}}$ of $1 \mathrm{~h}, C L / F$ of $1, V / F$ of $12 / 0.693, t_{1 / 2, k_{\mathrm{c}}}$ of $12 \mathrm{~h}$, and $t_{\max }$ of $2.821 \mathrm{~h}$ in the present study. The sampling time was assumed to be $3 \mathrm{~h}$ and $12 \mathrm{~h}$ after a repetitive twicedaily dose $(\tau=12 \mathrm{~h})$ for all 50 subjects, because we could not know the true $t_{\max }$ in individual subjects. Figure 2A shows the correlation between $C L / F_{\text {approx }, i}$ and $C L / F_{i}$ for a drug with $\omega_{C L / F}=\omega_{V / F}=0.4, \omega_{k_{2}}=0.8$, and $\sigma_{3}=\sigma_{12}=0.05$. Considerably large interindividual variability was assumed for $k_{\mathrm{a} i}, C L / F_{i}$, and $V / F_{i}$; however, the accuracy of $C L / F_{\text {approx, } i}$ was fairly good in all 50 subjects (Fig. 2A). Figure 3 shows the simulated blood drug concentration-time curve in 6 typical subjects, who are depicted with closed circles in Fig. 2A. We as-
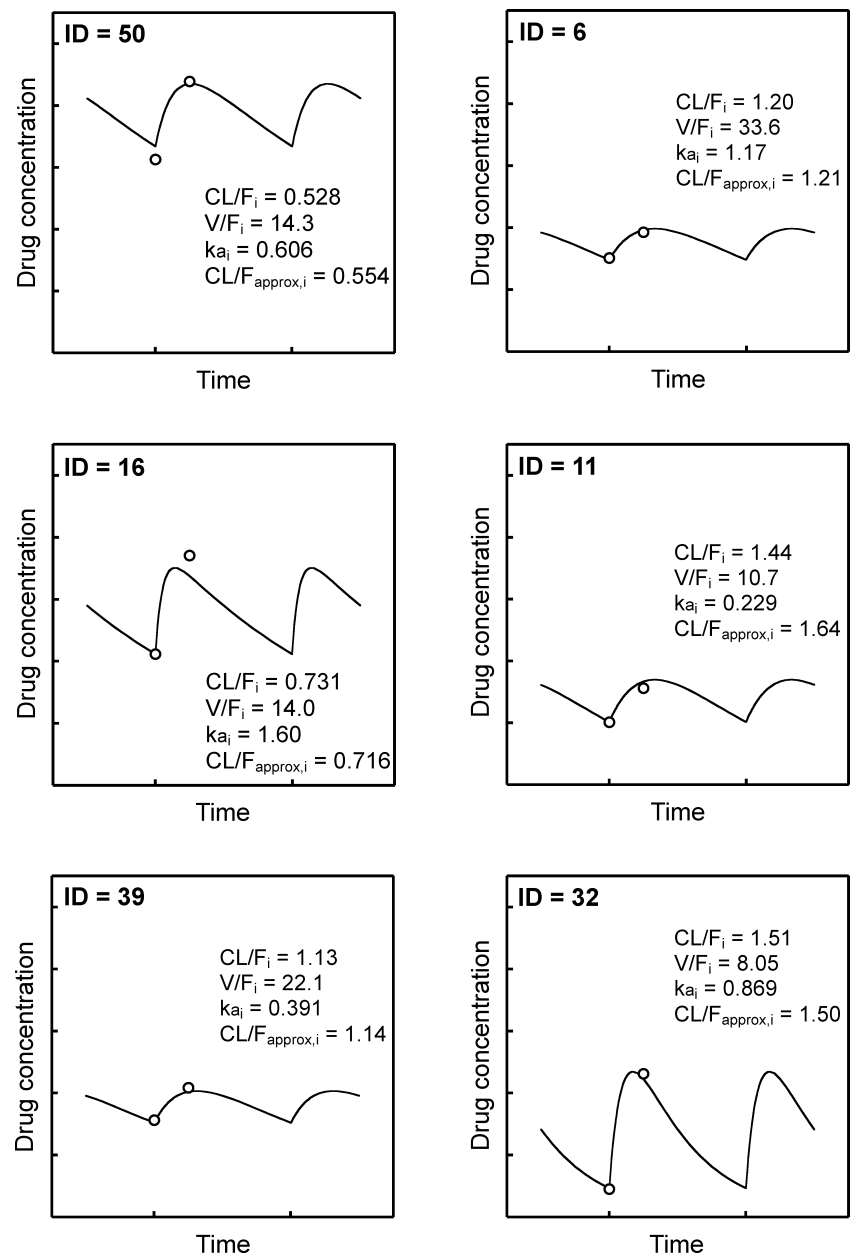

Fig. 3. Simulated Blood Drug Concentration-Time Curves in 6 Typical Subjects Shown in Fig. 2A

Open circles represent the measured blood concentration. 
sumed that we measured $C_{3 i}$ instead of $C_{\text {peak }}$ in each subject; nevertheless, $C_{3 i}$ seemed to be a good estimate of $C_{\text {peak }}$ in each subject (Fig. 3). In addition, $C L / F_{\text {approx }, i}$ was nearly equal to $C L / F_{i}$ even in the subject with considerably short $t_{1 / 2, k_{\mathrm{e}}}(\mathrm{ID}=32)$. These findings suggested that the accuracy of $C L / F_{\text {approx }, i}$ is fairly good even for drugs with large $\omega$ values when the measurement error of drug concentration (or intraindividual pharmacokinetic variability) is small.

It is readily conceivable that the large measurement error of drug concentration deteriorates the accuracy of $C L / F_{\text {approx }, i}$ significantly. The estimation error of $C L / F_{\text {approx }, i}$ was only marginally increased for a drug with $\sigma_{3}=\sigma_{12}=0.1$ as compared with a drug with $\sigma_{3}=\sigma_{12}=0.05$ (Figs. 2A, B), whereas it was considerably large for drugs with $\sigma_{3}=\sigma_{12}=0.15$ and $\sigma_{3}=\sigma_{12}=0.2$ (Figs. 2C, D). It should be noted that we may not estimate the $C L / F_{\text {approx }, i}$ value for drugs with large $\sigma$ values without multiple drug assays and/or blood sampling to obtain reliable mean values of $C_{\text {peak }}$ and $C_{\text {trough }}$, although it is still unclear whether the $\sigma$ value of 0.2 is realistic. ${ }^{8}$ In addition, the further decrease/increase in $\omega$ values seemed not to induce significant alterations of the accuracy of $C L / F_{\text {approx }, i}$ (Figs. 2E, F).

We performed additional computer simulations to mimic statistical hypothesis testing following clinical repeated-dose pharmacokinetic trials. The hypothetical drugs, dosing regimen, and sampling design were the same as those shown in Figs. 2 and 3 . In addition, the $C L / F$ value of the drug was assumed to be decreased to 0.7 by another concomitantly administered drug, or to be altered by the polymorphisms of drug-metabolizing enzymes. Table 3 shows the protocols and results of various in-silico clinical pharmacokinetic trials, where the number of replications of each trial was 30 in the present study. The statistical power to detect the difference of oral clearance between two groups was considerably high for a drug with $\omega_{C L / F}=\omega_{V / F}=0.4, \omega_{k_{\mathrm{a}}}=0.8$, and $\sigma_{3}=\sigma_{12}=0.1$, when the number of subjects in each group was set to 20 (Trial 1 in Table 3). The statistical power can be dependent on the magnitude of the measurement error of drug concentration because of the subsequent estimation error of $C L / F_{\text {approx }, i}$. Indeed, statistical significance following the $t$-test was marginally decreased for drugs with $\sigma_{3}=\sigma_{12}=0.15$ and $\sigma_{3}=\sigma_{12}=0.2$ (Trials 2, 3). On the other hand, the interindividual pharmacokinetic variability of drugs significantly affected the statistical power to detect the difference of oral clearance between two groups (Trials 4, 5). An unbalanced number of subjects in each group markedly affected the statistical significance (Trials 6,7). This simple experimental strategy may also be useful for designing the protocol of real clinical pharmacokinetic trials.

It should be noted that we did not extend the simulation to systematic evaluation of accuracy of $C L / F_{\text {approx }}$ for drugs with absorption lag time and/or with biexponential disposition in the present study, because accuracy of the estimated $C L / F$ value should be dependent not only on the pharmacokinetic characteristics of drugs (mean pharmacokinetic parameters and inter- and intraindividual variability), but also on study protocols/designs ( $\tau$ and sampling time). Eventually, the accuracy and precision of the analysis method to estimate oral clearance $\left(C L / F_{\text {approx }}\right)$ for a target drug of individual researchers should be evaluated carefully prior to a real clinical trial.

In conclusion, the present simulation study suggested that the peak-and-trough sampling design to estimate the $C L / F_{\text {approx }}$ value is useful for clinical repeated-dose pharmacokinetic trials, and that the study design and protocol should be evaluated carefully by computer simulation prior to a real clinical trial.

Acknowledgement This work was supported in part by a Grant-in-Aid for Scientific Research from the Japan Society for the Promotion of Sciences (JSPS).

\section{REFERENCES}

1) Hashimoto Y., Sheiner L. B., J. Pharmacokinet. Biopharm., 19, 333353 (1991).

2) Taguchi M., Nozawa T., Mizukami A., Inoue H., Tahara K., Takesono C., Hashimoto Y., Biol. Pharm. Bull., 27, 1642-1648 (2004).

3) Taguchi M., Nozawa T., Igawa A., Inoue H., Takesono C., Tahara K., Hashimoto Y., Biol. Pharm. Bull., 28, 876-871 (2005).

4) Taguchi M., Fujiki A., Iwamoto J., Inoue H., Tahara K., Saigusa K., Horiuchi I., Oshima Y., Hashimoto Y., Biol. Pharm. Bull., 29, 517521 (2006).

5) Honda M., Ogura Y., Toyoda W., Taguchi M., Nozawa T., Inoue H., Hashimoto Y., Biol. Pharm. Bull., 29, 772-778 (2006).

6) Honda M., Itoh H., Suzuki T., Hashimoto Y., Biol. Pharm. Bull., 29, 2460-2464 (2006).

7) Koue T., Kubo M., Funaki T., Fukuda T., Azuma J., Takaai M., Kayano Y., Hashimoto Y., Biol. Pharm. Bull., 30, 2154-2158 (2007).

8) Sheiner L. B., Beal S. L., J. Pharmacokinet. Biopharm., 11, 303-319 (1983). 